\title{
Review of Forward Physics at RHIC
}

\author{
R. Debbe for the BRAHMS Collaboration \\ Brookhaven National Laboratory, Upton, N.Y. 11973 USA \\ Presented at the Strong \& Electroweak Matter 2006 International Conference \\ Upton, New York \\ May 10-13, 2006 \\ Published by Elsevier Science Publisher in Nuclear Physics A
}

August 2006

\section{Physics Department \\ Brookhaven National Laboratory \\ P.O. Box 5000 \\ Upton, NY 11973-5000 \\ www.bnl.gov}

Notice: This manuscript has been authored by employees of Brookhaven Science Associates, LLC under Contract No. DE-AC02-98CH10886 with the U.S. Department of Energy. The publisher by accepting the manuscript for publication acknowledges that the United States Government retains a non-exclusive, paid-up, irrevocable, world-wide license to publish or reproduce the published form of this manuscript, or allow others to do so, for United States Government purposes. 


\section{DISCLAIMER}

This report was prepared as an account of work sponsored by an agency of the United States Government. Neither the United States Government nor any agency thereof, nor any of their employees, nor any of their contractors, subcontractors, or their employees, makes any warranty, express or implied, or assumes any legal liability or responsibility for the accuracy, completeness, or any third party's use or the results of such use of any information, apparatus, product, or process disclosed, or represents that its use would not infringe privately owned rights. Reference herein to any specific commercial product, process, or service by trade name, trademark, manufacturer, or otherwise, does not necessarily constitute or imply its endorsement, recommendation, or favoring by the United States Government or any agency thereof or its contractors or subcontractors. The views and opinions of authors expressed herein do not necessarily state or reflect those of the United States Government or any agency thereof. 


\title{
Review of Forward Physics at RHIC
}

R. Debbe ${ }^{\mathrm{a}}$

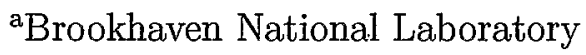

The RHIC high energy collision of species ranging from $p+p, p(d)+A$ to $A+A$ provide access to the small-x component of the hadron wave function. The RHIC program has brought renewed interest in that subject with its ability to reach values of the parton momentum fraction smaller than 0.01 with studies of particle production at high rapidity. Furthermore, the use of heavy nuclei in the $\mathrm{p}(\mathrm{d})+\mathrm{A}$ collisions facilitates the study of saturation effects in the gluonic component of the nuclei because the appropriate scale for that regime grows as $A^{\frac{1}{3}}$. We review the experimental results of the RHIC program that have relevance to small- $x$ emphasizing the physics extracted from $\mathrm{d}+\mathrm{Au}$ collisions and their comparison to $\mathrm{p}+\mathrm{p}$ collisions at the same energy.

\section{INTRODUCTION}

Hadronic interactions in colliders have attained the highest energies up to date. The cross-sections of identified pions produced in $\mathrm{p}+\mathrm{p}$ collisions at RHIC $(\sqrt{s}=200 \mathrm{GeV})$ are now well described by Next-to-Leading-Order perturbative Quantum Chromo Dynamics (NLO pQCD) calculations at mid and forward rapidities, as can be seen in Fig. 1. This success of QCD allows us to confidently describe the RHIC measurements above $1-2 \mathrm{GeV} / \mathrm{c}$ in $p_{T}$ as related to sufficiently hard interactions that can be calculated with perturbative techniques and partonic degrees of freedom. The study of high energy interactions in collider mode opens access to an extended reach in rapidity space and facilitates the study of hadrons wave functions at both extremes of the fractional longitudinal momentum $x \sim 0$ and $x \sim 1$. Because the study of particle production at high values of rapidity skews the kinematics of the interaction at the partonic level in such a way, that one of interacting partons has a small momentum fraction $\left(x \leq 10^{-3}\right.$ around $y=3$ at RHIC), while the second one carries most of the beam momentum.

\section{BRAHMS}

The Forward Spectrometer (FS) of the BRHAMS Collaboration is particularly well suited to study charged particle production at high rapidity. The complement of tracking, Cerenkov's ring imaging and time-of-flight techniques have been used to extract new physics from the comparison of invariant yields of charged particles in $\mathrm{d}+\mathrm{Au}$ and $\mathrm{p}+\mathrm{p}$ collisions at the same energy. Such comparison is shown in Fig. 2 with the so called nuclear modification factor $R_{d A u}$ [5]. The changes in magnitude and shape of the $R_{d A u}$ factor seen in this figure as rapidity values grow from mid-rapidity $(\eta=0)$ on the left- 


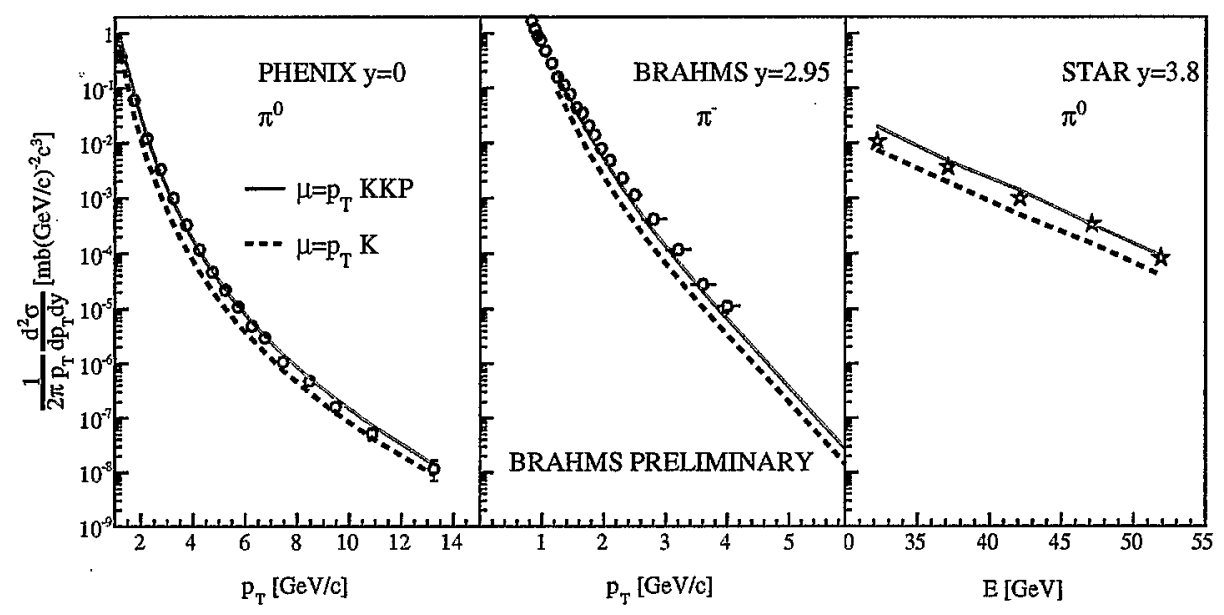

Figure 1. Invariant cross sections for pion production in $\mathrm{p}+\mathrm{p}$ collisions at RHIC $\sqrt{s}=$ $200 \mathrm{GeV}$ The left-most panel shows the neutral pion distribution measured by PHENIX [1] at mid-rapidity as function of transverse momentum. The central panel shows the BRAHMS measurement of negative pions at $y=2.95$ as function of transverse momentum, and the right-most panel shows the neutral pions measured by the STAR collaboration [2] as function of the pion energy. In all three panels the NLO pQCD calculations are shown as smooth curves at one scaling parameter $\left(\mu=p_{T}\right)$ and two sets of fragmentation functions KKP [3] and K [4]. All three measurements favor the KKP set of fragmentation functions indicating a strong contribution from gluon-quark and gluon-gluon interactions. All errors shown in the figures are statistical.

most panel all the way to the highest value $(\eta=3.2)$ shown on the right-most panel, are consistent with the onset of the Color Glass Condensate (CGC) [10] at RHIC.

The CGC is expected to appear as a form of high density gluonic matter as the momentum fraction of the partons tends to zero. In a CGC based description of $\mathrm{d}+\mathrm{Au}$ collisions, the partons in the Au wave function tend to populate states with transverse momentum values around the saturation scale $Q_{s}$ and the valence quarks from the deuteron beam multiple scatter on them producing the enhancement seen in Fig. 2 at $\eta=0$. Away from $\eta=0$, the Au wave function is further modified by gluon emission (the probability of gluon emission per unit of rapidity is proportional to $\alpha_{s}$ the strong coupling constant). Gluons with small values of $x$ are delocalized and the gluon fields extend into other nucleons. Besides that, gluons have as well a strong tendency to fuse and the evolution of the wave function with rapidity ends up as a compromise between gluon emission and gluon fusion that tends toward a limiting value. The comparison between $d+A u$ and $p+p$ yields shown with the $R_{d A U}$ ratio will show an stronger suppression as rapidity grows because the numerator would be reaching the above mentioned limiting value, while the denominator is still growing with rapidity because that system is considered dilute and is not affected by the small contribution from gluon fusion [11,12]. Such behavior can be seen in the measured rapidity dependence of the $R_{d A u}$ factor displayed in Fig. 2. The 


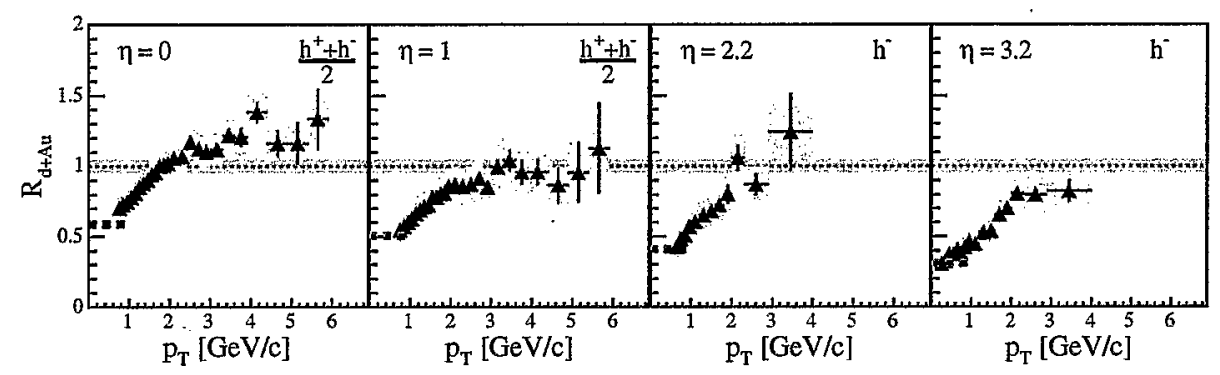

Figure 2. Nuclear modification factor extracted from minimum biased sample of charged particles at $\eta=0$ and $\eta=1$. The same factor, but this time for negative hadrons is shown in the two right-most panels for $\eta=2.2$ and $\eta=3.2$. The mean number of collisions used to make this factor is $7.2 \pm 0.3$. Statistical errors are shown with vertical lines and systematic errors with gray boxes.

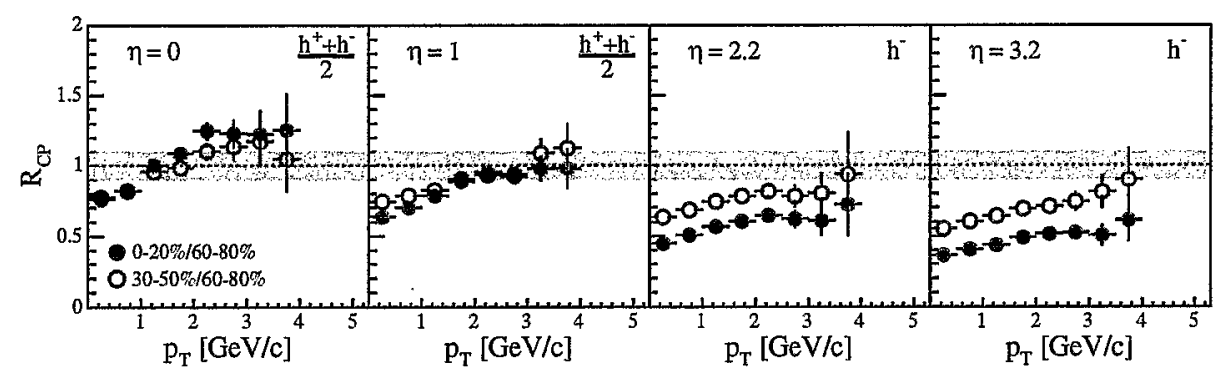

Figure 3. Nuclear modification factor extracted from the comparison of $d+$ Au data samples with different centralities at the same pseudo-rapidities as Fig. 2.

effects of quantum evolution are more pronounced in central events. Central events have the highest number of interacting nucleons that, as mentioned above, share their small- $x$ gluon fields increasing the chances for gluon fusion. These systems with higher number of gluons would reach the limiting value of their evolution with rapidity much earlier than the nuclei interacting with bigger impact parameters. Fig. $3 R_{c p}$ has been constructed with three data samples: central events with centralities in the 0-20\% range, mid-central events in the range $30-50 \%$ and the peripheral events used as reference with centralities in the $60-80 \%$ range. At mid-rapidity the central $R_{c p}$ points are systematicaly above the semi-central ones, but at the highest rapidity $(\eta=3.2)$, the trend is reversed, and this time the central events have a very much suppresed value of $R_{c p}$ compared to the semi-central sample. 


\section{PHOBOS}

The PHOBOS Collaboration has presented similar results as the ones shown in the previous section. They measure the yields of charged hadrons with their two-arm magnetic spectrometer equipped with high resolution silicon based tracker. They have extracted the nuclear modification factor from a centrality averaged data sample of charged hadrons in three pseudo-rapidity bins, $0.2<\eta<0.6,0.6<\eta<1.0$ and $1.0<\eta<1.4$. They use charged hadron spectra measured by the UA1 Collaboration [7] in $p+\bar{p}$ at the same energy, and a suitable correction is applied to account for the difference in acceptance between the two experiments.

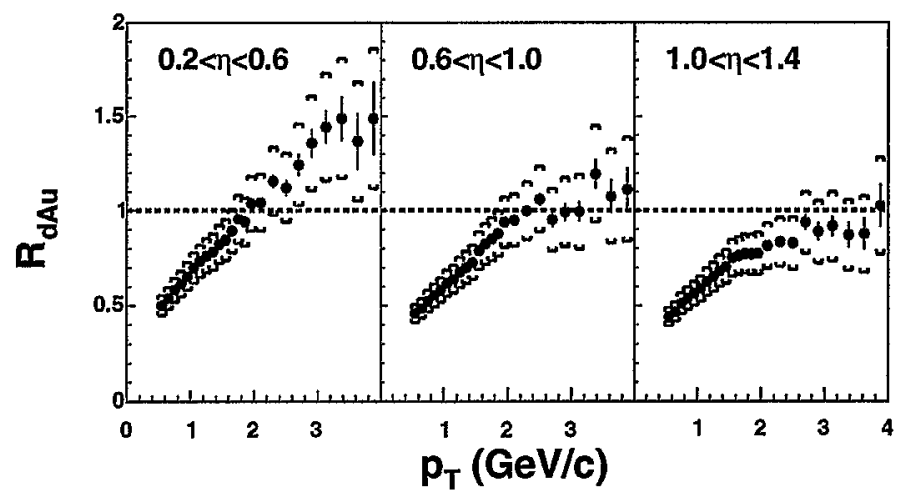

Figure 4. Nuclear Modification factor $R_{d A u}$ for charged hadrons measured in the PHOBOS two-arm spectrometer as function of the transverse momentum in three pseudo-rapidity bins. The brackets show the systematic errors.

Figure 4 shows the nuclear modification factor as a function of the transverse momentum, The mean number of collisions used to construct this factor is equal to $9.5 \pm$ 0.8 (systematic). The remarkable change in the shape of the $R_{d A u}$ factor as the pseudorapidity changes by only half a unit has been highlighted as an indication of the fast onset of the effects of quantum evolution on an already dense Au wave function.

\section{PHENIX}

The PHENIX collaboration has measured the yield of charged hadrons with their muon spectrometers covering the $\mathrm{Au}$ fragmentation region (backward) $-2.2<\eta<-1.2$, and the deuteron fragmentation $1.2<\eta<2.2$ (forward). Although these spectrometers are optimized to detect muons, they can also be used to detect charged particles that penetrate the spectrometer up to its last active stages. These penetrating particles are designated as "punch-through hadrons" (PTH). A second method detects the muons from 
hadrons that decay before the first layer of absorber in the spectrometer, these particles are referred as "hadron decay muons" (HDM) [8].

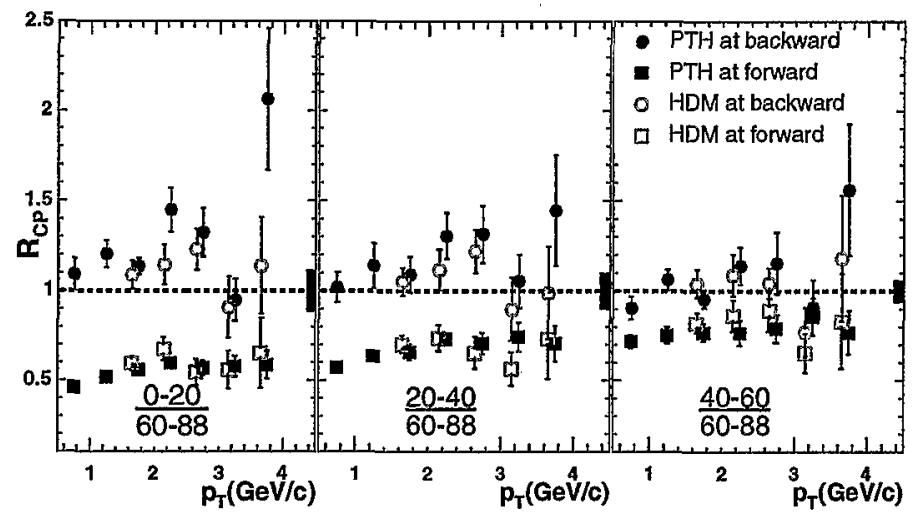

Figure 5. Nuclear modification factor $R_{c p}$ as function of $p_{T}$ in the forward (squares) and backward rapidity (circles). These factors are extracted from four data samples with different centralities. The centrality of the event is measured with the backward BBC detectors.

Figure 5 shows, first of all, the consistent results obtained with the PTH and HDM techniques. The measurement performed on the Au fragmentation side (backward) shows a weak enhancement that is most pronounced in the most central sample $\left(\frac{0-20 \%}{60-88 \%}\right)$. A strong suppression on the deuteron fragmentation side is seen in the central event sample. Such suppression is reduced as the centrality selection changes in the $\left(\frac{40-60 \%}{60-88 \%}\right)$ ratio. The PHENIX forward factors are consistent with the ones measured by BRAHMS and PHOBOS. The backward measurements probe intermediate values of the momentum fraction $x$ in the Au wave function, the hint of an enhancement remains unexplained and it could be a combination of different effects [8].

Figure 6 shows the integrated $R_{c p}$ distributions over the intervall $1.5<p_{T}<4.0 \mathrm{GeV} / \mathrm{c}$ at different values of pseudo-rapidity within the acceptance of both muon spectrometers. The main feature on this figure is the strong suppression in the forward side (deuteron fragmentation) for the most central events, as well as a clear enhancement in the Au fragmentation side for the same data sample.

\section{STAR}

The STAR collaboration has measured the yield of high energy neutral pions $(25<$ $\left.E_{\pi}<55 \mathrm{GeV}\right)$ at high rapidity $(3.0 \leq \eta \leq 4.2)$ in $\mathrm{p}+\mathrm{p}$ and $\mathrm{d}+\mathrm{Au}$ collisions at $200 \mathrm{GeV}$ with their forward $\pi^{0}$ detector (FPD) [9]. Figure 7 shows the nuclear modification factor $R_{d A u}$ for neutral pions (filled circles) at $\langle\eta\rangle=4$, the highest rapidity studied at RHIC so far. The figure also shows the BRAHMS factors extracted at $\eta=2.2$ (open circles), 


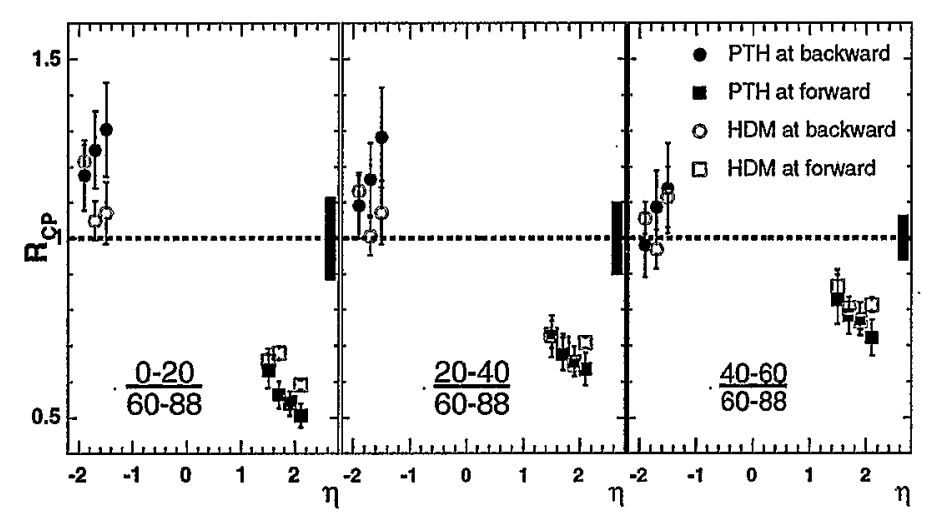

Figure 6. The integrated Nuclear modification factor $R_{c p}$ as function of pseudo-rapidity measured in both the Au side (backward) and the deuteron side (forward) extracted from four different centrality data samples.

and $\eta=3.2$ (open squares). Both BRAHMS and STAR data were extracted without conditions on centrality. The suppression seen in the STAR $\pi^{0}$ factor is stronger than the one measured with negative hadrons and both measurements are consistent if the isospin effects in $\mathrm{p}+\mathrm{p}$ are taken into account as well as the higher rapidity value of the STAR measurement. The inset shows the STAR $R_{d A u}$ factor together with ratios built from NLO pQCD calculations with the KKP and $K$ sets of fragmentation functions and a third calculation that includes coherent multiple scattering in the Au target [15]. The suppression found in the data is clearly much stronger than the calculations. For more details please refer to [9].

STAR can correlate high energy neutral pions at high rapidity and charged hadrons detected in the Time-Projection-Chamber (TPC). The difference in azimuth angle between the forward neutral pion, and charged particles detected in the TPC $(|\eta|<0.75)$ with transverse momentum greater than $0.5 \mathrm{GeV} / \mathrm{c}$ is shown in Fig. 8. Azimuth angle correlations measured in $\mathrm{p}+\mathrm{p}$ collisions are shown in the left column of the figure and the ones extracted from $\mathrm{d}+\mathrm{Au}$ events are shown on the right column. The effects of gluon emission between the parton that generates the forward pion and the one related to the charged particle detected near mid-rapidity would destroy the back-to-back correlation and would appear as the production of mono-jets [13]. Such effect on the azimuthal angle correlation is visible in the low pion energy bin, but is not present in the second, higher energy bin. This result is tantalizing and has been presented as another indication of the onset of coherence in the CGC. The PHENIX collaboration has extracted similar correlations and they found no indication of de-correlation of back-to-back jets. The PHENIX measurement was performed with hadrons separated by a smaller rapidity gap than the STAR measurement [14]. Further studies of this nature are expected to be performed in near future RHIC runs.

Other theoretical approaches have been offered to explain the suppression at high ra- 


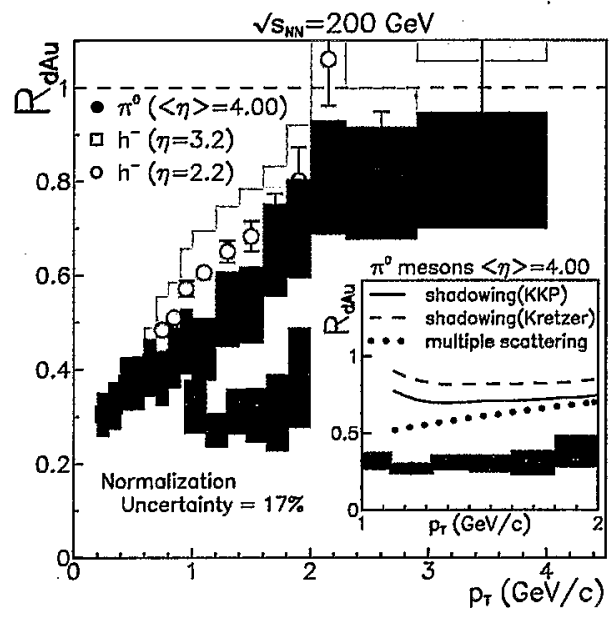

Figure 7. STAR nuclear modification factor $\left(R_{\mathrm{dAu}}\right)$ for minimum-bias neutral pion production in $\mathrm{d}+\mathrm{Au}$ collisions versus transverse momentum $\left(p_{T}\right)$, shown with filled circles. The BRAHMS factors extracted for negative hadrons smaller at $\eta=2.2$ and $\eta=3.2$ are also shown with open circles and boxes respectively [5]. The error bars are statistical, and the shaded boxes show the point-to-point systematic errors. (Inset) The STAR $R_{\mathrm{dAu}}$ compared to the ratio of NLO pQCD calculations and [15].

pidity in $d+A u$ collisions. Such is the case of a description of $d+A$ collision as coherent scattering equivalent to a shift in $\mathrm{x}[15]$. Perturbative $\mathrm{QCD}$ calculations with a standard shadowing parametrization added to the parton distribution functions [16] are also available. Other calculations emphasize the effects of parton recombination [17], and finally, one of the latest explanations to the high rapidity suppression focuses on energy conservation arguments that may be applicable for deuteron valence quarks with high $x$ values [18]

\section{FUTURE PROSPECTS}

Two of the RHIC experiments (BRAHMS and PHOBOS) have by now ended their data taking phase, and the STAR and PHENIX collaborations are well into a detector upgrade program aimed to increase their rapidity coverage, and to enhance their ability to detect rare processes connected to heavier quarks. These upgrades are scheduled to come on-line as RHIC evolves into its second phase when it will provide collisions at higher luminosity. The STAR collaboration is working on a bigger Lead-glass array called the Forward Meson Spectrometer (FMS) placed at the same location of the existing FPD on the detuteron fragmentation side. This detector will have a rapidity coverage of $2.5<\eta<4.0$. The FMS will be used to continue the study of correlations between high energy mesons and photons and charged particles detected with the TPC in search for the onset of saturation in possible "macroscopic gluons fields" [19].

The PHENIX collaboration is adding precision tracking before the first muon absorber 


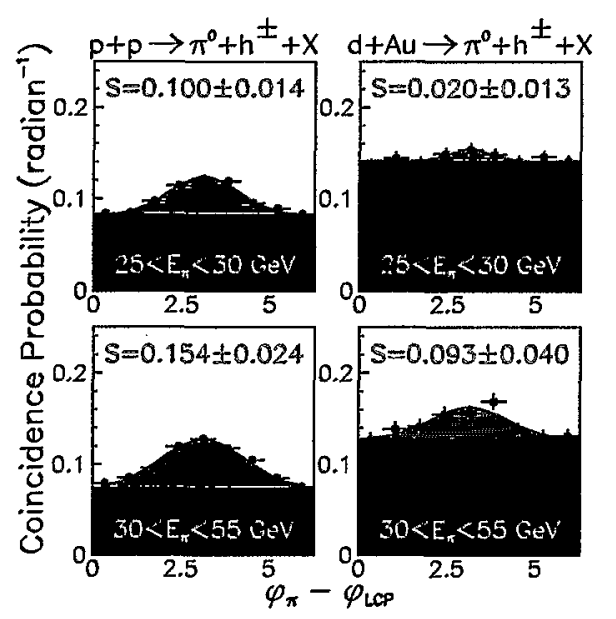

Figure 8. Azimuthal correlations centered around $\Delta \phi=\pi$ between a forward high energy neutral pion and charged hadrons detected around mid-rapidity in the STAR TPC. The left column shows the correlation obtained from $\mathrm{p}+\mathrm{p}$ events at two pion energy bins: 25. $<E_{\pi}<30 \mathrm{GeV}$ (top) and 30. $<E_{\pi}<55 \mathrm{GeV}$ (bottom). The right column shows the same correlation extracted from $\mathrm{d}+\mathrm{Au}$ collisions. The errors are statistical. For further details see [9].

with the Forward Silicon Tracker. They will also replace the "nose-cone" hadronic absorbers in the muon arms with a highly segmented Si-W calorimeters dubbed Nose Cone Calorimeter (NCC). This addition to the PHENIX detector will extend its pseudo-rapidity coverage up to a value of 3 to allow for precision measurements of direct photons, $\pi^{0} \mathrm{~s}$ and di-electrons in $\mathrm{A}+\mathrm{A}, \mathrm{p}(\mathrm{d})+\mathrm{A}$, and polarized $\mathrm{p}+\mathrm{p}$ collisions, in particular it will be able to identify pions with momentum as high as $30 \mathrm{GeV} / \mathrm{c}$. Together with the Forward Silicon Tracker, the NCC can be used to measure D and B meson productions via their semi-leptonic decays.

\section{SUMMARY}

The study of the RHIC d+Au collisions, together with results extracted from the several $\mathrm{p}+\mathrm{p}$ RHIC runs, have renewed the interest in the small-x physics of hadronic systems. Together with the lower than expected multiplicities detected in $\mathrm{A}+\mathrm{A}$ collisions at top RHIC energies, these results indicate the onset of a saturated regime that exhibits coherence. Further studies of $d+A u$ collisions at high rapidity are planed for the near future by the two big RHIC experiments. These measurements will include a search of mono-jets as well and a shift to probes that have a more direct connection to the gluon component of the Au nuclei such as direct virtual and real photon production as well as open charm detection at high rapidity . 


\section{REFERENCES}

1. S. S. Adler, et al., Phys. Rev. Lett. 91, 241803 (2003).

2. J. Adams, et al., Phys. Rev. Lett. 92, 171801 (2004).

3. B.A. Kniehl et al., Nucl. Phys. B597, 337 (2001).

4. S. Kretzer et al, Eur. Phys. J. C22, 269 (2001).

5. I. Arsene et al., Phys. Rev. Lett. 93, 242303, (2004).

6. B.B. Back et al., Phys. Rev. C70, 061901, (2004).

7. C. Albajar et al., Nucl. Phys. B335, 261 (1990).

8. S. S. Adler, et al., Phys. Rev. Lett. 94, 082302 (2005).

9. J. Adams et al., nucl-ex/0602011.

10. L. McLerran and R. Venugopalan, Phys. Rev. D 49, 3352 (1994);

11. R. Baier, A. Kovner and U. Wiedemann Phys. Rev. D68, 054009 (2003).

12. D. Kharzeev, Y. Kovchegov and K. Tuchin Phys. Rev. D68, 094013 (2003).

13. D. Kharzeev, Nucl. Phys. A 715, 35c (2003).

14. S. S. Adler, et al., Phys. Rev. Lett. 96, 222301 (2006).

15. J. Qiu and I. Vitev, Phys. Rev. Lett. 93, 262301 (2004); hep-ph/0410218.

16. R. Vogt, Phys. Rev. C 70, 064902 (2004); V. Guzey, M. Strikman, and W. Vogelsang, Phys. Lett. B 603, 173 (2004).

17. R. C. Hwa, C. B. Yang, and R. J. Fries, Phys. Rev. C 71, 024902 (2005).

18. B. Kopeliovich et al, Phys. Rev. C 72, 054606 (2005);

19. L. C. Bland, et al., Eur. Phys. J. C 43, 427 (2005). 